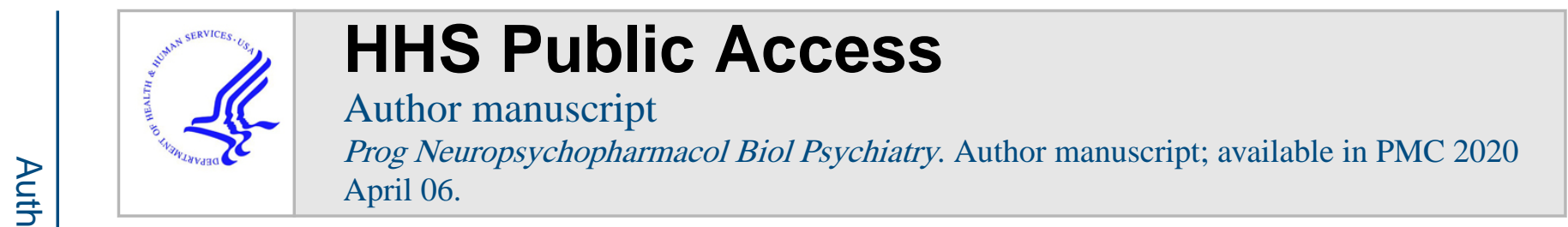

Published in final edited form as:

Prog Neuropsychopharmacol Biol Psychiatry. 2014 June 03; 51: 99-104. doi:10.1016/

j.pnpbp.2014.01.012.

\title{
The BDNF Val66Met polymorphism and plasma brain-derived neurotrophic factor levels in Han Chinese patients with bipolar disorder and schizophrenia
}

\author{
Shiou-Lan Chen ${ }^{\# 2,7,{ }^{*} \text {, Sheng-Yu Lee }}{ }^{2,3,5}$, Yun-Hsuan Chang ${ }^{2,4}$, Shih-Heng Chen ${ }^{2,8}$, Chun- \\ Hsien Chu ${ }^{2,8}$, Tzu-Yun Wang ${ }^{2,3,6}$, Po-See Chen ${ }^{2,3,5}$, I-Hui Lee ${ }^{2,3,5}$, Yen-Kuang Yang ${ }^{2,3,5}$, Jau- \\ Shyong Hong ${ }^{8}$, Ru-Band Lü ${ }^{\# 1,2,3,4,5,{ }^{*}}$ \\ ${ }^{1}$ Institute of Behavioral Medicine, National Cheng Kung University; \\ 2Department of Psychiatry, National Cheng Kung University \\ ${ }^{3}$ Hospital, College of Medicine, National Cheng Kung University; \\ ${ }^{4}$ Institute of Allied Health Sciences, College of Medicine, National Cheng Kung University; \\ ${ }^{5}$ Addiction Research Center, National Cheng Kung University; \\ ${ }^{6}$ Department of Psychiatry, Tainan Hospital, Department of Health, Executive Yuan, Tainan; \\ ${ }^{7}$ Department of Neurology, School of Medicine, Kaohsiung Medical University, Kaohsiung, \\ Taiwan; \\ ${ }^{8}$ Neuropharmacology Section, Laboratory of Pharmacology and Chemistry, National Institute of \\ Environmental Health Sciences/National Institutes of Health, Research Triangle Park, North \\ Carolina, USA \\ \# These authors contributed equally to this work.
}

\section{Abstract}

Objective-Brain-derived neurotropic factor (BDNF) is widely distributed in the peripheral and central nervous systems. BDNF and its gene polymorphism may be important in synaptic plasticity and neuron survival, and may become a key target in the physiopathology of several mental illnesses. To elucidate the role of BDNF, we compared the plasma BDNF levels and the BDNF Val66Met gene variants effect in several mental disorders.

\footnotetext{
*Corresponding Authors: Assistant Professor, Shiou-Lan Chen, PhD, Department of Psychiatry, College of Medicine, National Cheng Kung University, 138 Sheng-Li Road, Tainan 70428, Taiwan, Tel: +886-6-235-3535 ext. 5836; Fax: +886-6-302-8012; shioulan@mail.ncku.edu.tw; Professor Ru-Band Lu, MD, Department of Psychiatry, College of Medicine and Hospital, National Cheng Kung University, 138 Sheng-Li Road, Tainan 70428, Taiwan, Tel: +886-6-235-3535 ext. 5108; Fax: +886-6-302-8012; rblu@mail.ncku.edu.tw; rubandlu@gmail.com.

Author Contributions

SLC and RBL wrote the first draft. SYL, YHC, SHC and CHC managed the lab work and statistics. TYW, PSC, IHL, TLY and YKY managed the participant recruitment. JSH supervised this work and edited the manuscript.

Publisher's Disclaimer: This is a PDF file of an unedited manuscript that has been accepted for publication. As a service to our customers we are providing this early version of the manuscript. The manuscript will undergo copyediting, typesetting, and review of the resulting proof before it is published in its final form. Please note that during the production process errors may be discovered which could affect the content, and all legal disclaimers that apply to the journal pertain.

Conflict of interest

All of the authors declared no conflicts of interest.
} 
Method-We enrolled 644 participants: 177 patients with bipolar I disorder (BP-I), 190 with bipolar II disorder (BP-II), 151 with schizophrenia, and 126 healthy controls. Their plasma BDNF levels and $B D N F$ Val66Met single nucleotide polymorphisms (SNP) were checked before pharmacological treatment.

Results-Plasma levels of BDNF were significantly lower in patients with schizophrenia than in healthy controls and patients with bipolar disorder $(\mathrm{F}=37.667, \mathrm{p}<0.001)$; the distribution of the $B D N F$ Val66Met SNP was not different between groups $\left(\chi^{2}=5.289, \mathrm{p}=0.507\right)$. Nor were plasma BDNF levels significantly different between Met/Met, Met/Val, and Val/Val carriers in each group, which indicated that the BDNF Val66Met SNP did not influence plasma BDNF levels in our participants. Plasma BDNF levels were, however, significantly negatively correlated with depression scores in patients with bipolar disorder and with negative symptoms in patients with schizophrenia.

Conclusion-We conclude that plasma BDNF profiles in different mental disorders are not affected by $B D N F$ Val66Met gene variants, but by the process and progression of the illness itself.

\section{Keywords}

BDNF Val66Met gene; bipolar disorders; plasma BDNF; schizophrenia

\section{Introduction}

Brain-derived neurotropic factor (BDNF) is a member of the nerve growth factor family, which is involved in neuron survival and differentiation (Mizuno et al., 1994), synaptogenesis, and maintenance (Ohira and Hayashi, 2009). BDNF is a basic dimeric protein that has to be maintained throughout life to preserve essential functions such as learning and memory. In humans, BDNF is expressed in the postnatal brain, with the highest mRNA levels in the hippocampus and cortex (Webster et al., 2002). BDNF promotes the survival of a wide range of neurons, which suggests that BDNF might be a biomarker for the pathophysiology of mental illness. For instance, the effects of BDNF on dopaminergic neurons might be associated with the pathogenesis of schizophrenia, which is apparently related to dopaminergic dysfunction (Hyman et al., 1991). Moreover, because BDNF acts on the primary sensory and cholinergic neurons of the basal forebrain (Alderson et al., 1990), its dysfunction might also be related to mood disorders and schizophrenia (Dilsaver, 1986, Berman et al., 2007). Thus, the plasma brain BDNF levels in these mental illnesses have been studied. In manic and depressed patients with bipolar disorder, serum BDNF levels are significantly lower than normal (Cunha et al., 2006, Monteleone et al., 2008). Low serum BDNF levels during acute manic and depressive episodes recover to levels similar to those of controls after patients have been treated and return to a euthymic state. BDNF levels in euthymic also decrease with age and the length of the illness (Fernandes et al., 2011). In patients with schizophrenia, BDNF data are inconsistent. Some studies show BDNF levels significantly lower than normal in serum (Toyooka et al., 2002) and related brain areas (Iritani et al., 2003, Weickert et al., 2003), but others show unaltered (Shimizu et al., 2003) or higher than normal serum BDNF levels in patients with schizophrenia (Gama et al., 2007). Furthermore, postmortem patients with schizophrenia also have elevated BDNF levels in the cortex and hippocampus (Takahashi et al., 2000). However, because of a dearth 
of published reports, small sample sizes, and inconsistent results, the role of BDNF in mental illness requires additional study.

In contrast, a number of genetic association studies have shown that a single nucleotide polymorphism (SNP) in the promoter of the $B D N F$ gene at codon 66 (G196A, rs6265) is associated with mental illness. The SNP in the $B D N F$ gene leads to a valine (Val) to methionine (Met) substitution at codon 66 in the pro domain (BDNFMet). This BDNF polymorphism (Val66Met) dramatically alters the intracellular trafficking and packaging of pro-BDNF, and reduces the trafficking and secretion of BDNF protein (Egan et al., 2003). In Caucasian patients with bipolar disorder, BDNFMet66 allele carriers are likely to have smaller regional brain volumes and more total cerebrospinal fluid (CSF) in the lateral ventricles than do Val66 homozygote carriers (Teh et al., 2012). A Val66Met polymorphism in the $B D N F$ gene and one in bipolar disorder have been tentatively associated (Fan and Sklar, 2008). In patients with schizophrenia, this Val66Met SNP in the $B D N F$ gene has also been associated with aggressive behavior (Spalletta et al., 2010), the executive function (Lu et al., 2012), obsessive-compulsive symptoms (Hashim et al., 2012), and prefrontal brain function (Whalley et al., 2010). One meta-analysis of case-control studies (Gratacos et al., 2007) found a relationship between the SNP and substance-related disorders, eating disorders, and schizophrenia. Another meta-analysis, however, found no association between the SNP and schizophrenia or bipolar disorder (Kanazawa et al., 2007). Thus, the role of the $B D N F$ Val66Met SNP in mental illness requires additional study. The inconsistent BDNF expression profiles might be attributable to the very small sample sizes of the studies on these mental disorders. In addition, correlations between different mental disorders and the $B D N F$ Val/Met SNP and plasma BDNF levels have not been reported. To clarify the plasma BDNF profile in human mental disorders, we included a large number of healthy controls and patients with bipolar I disorder, bipolar II disorder, and schizophrenia in the present study. Their plasma BDNF levels were analyzed and compared. The BDNFVal66Met SNP variant distribution in these participants was also studied to determine its association with these groups. Because the $B D N F$ Val66Met variant inhibits the trafficking and secretion of BDNF protein, we also studied its effect on the plasma BDNF protein levels in all participants.

\section{Experimental procedure}

\subsection{Participants}

From the Department of Psychiatry at National Cheng Kung University Hospital and the National Defense Medical Center, we enrolled 644 Taiwanese participants: 126 healthy controls, 177 with bipolar I disorder (BP-I), 190 with bipolar II disorder (BP-II), and 151 with schizophrenia. The Chinese version of the Schedule for Affective Disorders and Schizophrenia-Lifetime (SADS-L), which has good reliability and validity, was used to screen all participants' psychiatric conditions, to confirm that they fit DSM-IV diagnostic criteria, and to verify that the participants had no physical and mental comorbidities (Endicott and Spitzer, 1978, Huang et al., 2004).

The healthy control group included 126 volunteers recruited from the hospital staff and community. At first, we put up a notice in the hospitals and communities to recruit healthy 
volunteers. The volunteers were first screened by telephone. Subjects whom had sleep disorder, substance abuse, mood swing or possible psychiatric traits in life were first excluded. The remained volunteers were then carefully screened. The Chinese version of the SADS-L was used to screen the psychiatric conditions of the controls. All of them were free of present and past major and minor mental illnesses (affective disorder, schizophrenia, anxiety disorder, personality disorder, alcohol abuse/dependence, and illegal substance use disorders). Additionally, none of the first-degree relatives of the healthy controls had a history of psychiatric disorder.

Mood disorder diagnoses were made according to DSM-IV criteria, except that, for bipolar II disorder, the 4 day duration criterion for hypomania was replaced with a 2-day cutoff duration because the 4-day minimum might not be evidence-based (Dunner, P. Goodnick, Editor. 1998) and because the 2-day cutoff has been supported by several previous reports (Benazzi and Akiskal, 2006). The inclusion criteria for patients with BP were as follows: 1) being a psychiatric inpatient and or outpatient who sought psychiatric care or had been referred for psychiatric evaluation because they were clinically suspected of having a mood disorder; 2) a diagnosis of bipolar I or II disorder based on DSM-IV criteria and confirmed by the Chinese version of the modified SADS-L; 3) a man or woman between 18 and 65 years old; 4) a Hamilton Depression Rating Scale (HDRS) score of at least 18 or a Young Mania Rating Scale (YMRS) score of at least 14 at the screening stage; 5) drug naïve or at least for the medications normally prescribed for bipolar disorder; 6) could communicate in Mandarin Chinese or Taiwanese; and 7) an informed consent form signed by the patient or a legal representative.

Patients with schizophrenia were screened using the modified Chinese version of the SADSL. They were checked for comorbid psychiatric conditions and to verify that their diagnosis fit DSM-IV diagnostic criteria. The inclusion criteria for patients with schizophrenia were: 1) being a psychiatric inpatient and or outpatient who sought psychiatric care or had been referred for psychiatric evaluation because they were clinically suspected of having a psychosis symptom; 2) a diagnosis of schizophrenia based on DSM-IV criteria and confirmed by the Chinese version of the modified SADS-L; 3) a man or woman between 18 and 65 years old; 4) drug naïve or at least for the medications normally prescribed for schizophrenia); 5) could communicate in Mandarin Chinese or Taiwanese; and 6) an informed consent form signed by the patient or a legal representative.

The following were the exclusion criteria for patients with bipolar disorder and schizophrenia: 1) women who were pregnant or had childbearing potential under the specific physiological condition; 2) any major mental illness other than BP or schizophrenia, including alcoholism and illegal substance use disorder; 3) any clinically significant comorbid medical condition, e.g., cardiac, hepatic, or renal disease, with current evidence of poor control of that disease.

Study design-The research protocol was approved by the institutional review board for the Protection of Human Subjects at National Cheng Kung University Hospital and the National Defense Medical Center. To minimize the effect of ethnic differences on gene frequencies, only participants who were unrelated and came from the Han Chinese 
population in Taiwan were recruited. Before the participants signed a written informed consent form, all procedures were fully explained to them.

\subsection{Collecting and assessing blood samples}

Ten milliliters of whole blood was drawn from the antecubital vein of each study participant. Blood were collected in tubes with EDTA (ethylenediaminetetraacetic acid) for plasma preparation. Plasma was isolated from the whole blood after it had been centrifuged at 3000 $g$ for $15 \mathrm{~min}$ at $4^{\circ} \mathrm{C}$ and then immediately stored at $-80^{\circ} \mathrm{C}$. Plasma BDNF levels were quantified using enzyme-linked immunosorbent assays (ELISA). A kit (Quantikine Human Cytokine Kit; R\&D Systems) and an ELISA reader (SpectraMax-M2; Molecular Devices) were used to analyze plasma BDNF levels.

\subsection{Genotyping}

DNA was extracted from the venous blood using a salting method. The G196A (Val66Met) $B D N F$ gene polymorphism was examined using a polymerase chain reaction (PCR) plus restriction fragment length polymorphism analysis. The following primers were used:

Forward: 5'-AGA GGC TTG ACA TCA TTG GCT-3';

Reverse: 5'-CGT GTA CAA GTC TGC GTC CT-3'.

DNA fragments were amplified in an Eppendorf thermal cycler and then digested overnight by PmlI restriction endonuclease (MBI Fermentas, Inc., Thermo Fisher Scientific). The digestion products were separated on a $4 \%$ agarose gel and visualized using ethidium bromide staining. Band size was determined using a DNA Ladder (O’Range Ruler 50 bp; MBI Fermentas). The undigested PCR product was 113 bp (allele A). Allele G was comprised of digested bands of 78 and $35 \mathrm{bp}$.

\subsection{Data analysis and statistics}

One-way analysis of variance (ANOVA), and then the Tukey-Kramer Post-hoc test was used for statistical evaluations. A $\chi^{2}$ test was used for categorical variables. Data are means \pm standard deviation (SD). To determine whether the plasma BDNF levels were significantly correlated with the genotype and disease symptoms, linear regression models were used. SPSS 17.0 for Windows was used for statistical computations. Significance was set at $\mathrm{p}<$ 0.05 .

\section{Results}

There were no differences in demographic data (gender, age, and body weight) between the healthy controls and patients (Table 1). Patients with schizophrenia had significantly lower plasma BDNF levels than did healthy controls $[\mathrm{F}(3,644)=37.667, \mathrm{p}<0.0001]$ (Table 1). Patients with schizophrenia had the lowest plasma BDNF levels $(6.7 \pm 7.0 \mathrm{ng} / \mathrm{mL})$ and healthy controls had the highest $(17.3 \pm 9.1 \mathrm{ng} / \mathrm{mL})$; patients in both bipolar disorder groups had levels almost as high as did the controls: BP-I $=15.3 \pm 10.0$ and BP-II $=14.6 \pm 10.0$.

In the $B D N F$ Val66Met variant distribution analysis, there was no significant difference between these four groups $\left(\chi^{2}=5.289, p=0.507\right)$, nor was there a significant difference in 
$B D N F$ Met and Val allele distribution frequency between groups $\left(\chi^{2}=1.649, \mathrm{p}=0.648\right)$

(Table 2). We did a multivariate linear regression analysis of the correlation of plasma BDNF level with the $B D N F$ gene variants (Met/Met, Met/Val, Val/Val) and different diagnosis (healthy control, BPI, BPII and schizophrenia). Plasma BDNF level were significantly correlated to different diagnosis $(\beta=-0.328, p<0.0001)$ but not significantly correlated to $B D N F$ Val66Met carriers $(\beta=0.016, \mathrm{p}=0.665)$, which indicated the $B D N F$ Val66Met variant does not affect peripheral BDNF levels but the different diagnosis of mental illnesses does. In patients with schizophrenia, all types of BDNF Val66Met carriers had the lowest plasma BDNF levels (Table 3), which indicated that the progression of schizophrenia might affect plasma BDNF levels, but the BDNF Val66Met variant did not.

We further did a multivariate linear regression analysis of the correlation of plasma BDNF level with the clinical symptoms. In patients with bipolar disorder, plasma BDNF levels were significantly negative correlated with the HDRS scores $(\beta=-0.174, p=0.014)$, but not the YMRS scores $(\beta=-0.117, \mathrm{p}=0.096$ ) or with comorbidities (anxiety disorder, nicotine dependency, phobias, and impulsivity: $\beta=0.078, p=0.178$ ). In patients with schizophrenia, plasma BDNF levels were significantly negatively correlated with negative symptoms ( $\beta=$ $-0.336, p=0.047)$ but not with positive symptoms $(\beta=0.008, p=0.948)$, aggressive symptoms $(\beta=-0.083, p=0.412)$, or the sum of symptoms $(\beta=0.2, p=0.365)$.

\section{Discussion}

We found different plasma BDNF expression profiles in patients with bipolar disorder and those with schizophrenia. Patients with BP-I and BP-II had non significantly lower plasma BDNF levels than did healthy controls, but patients with schizophrenia had significantly lower levels than did the other three groups of patients. BDNF can cross the blood-brain barrier (Pan et al., 1998), and BDNF levels in the brain and plasma undergo similar changes during maturation and aging in rats (Karege et al., 2002) and rhesus macaques (Cirulli et al., 2009). Moreover, lower plasma BDNF protein levels in humans might reflect the degree of neuronal degeneration in Alzheimer's disease (Laske et al., 2006) and age-related white matter atrophy in adult humans (Driscoll et al., 2012). These findings suggest that plasma BDNF levels might reflect BDNF levels in the brain, and that they might indicate the severity of mental disorders and the degree of a patient's neuronal degeneration.

\subsection{BDNF levels and bipolar disorder}

Although plasma BDNF levels in BP patients were not significantly lower than controls. However, they tended to be lowered than the healthy control and were significantly negatively correlated with the HDRS score, which indicated that they might influence the depressive behavior of patients with bipolar disorder. Previous study reported that serum BDNF levels were associated with depression-related personality trait in the general population of Caucasian people (Terracciano et al., 2011). Moreover, studies have reported that serum BDNF is lower in patients with BP during their manic and depressive episodes (Cunha et al., 2006, Monteleone et al., 2008). Peripheral levels (serum and plasma) of BDNF were lower in patients with depression and significantly increased after antidepressant treatment (Brunoni et al., 2008). These findings support the hypothesis of 
peripheral BDNF as a possible biological marker for depressive symptom in bipolar disorder. Agents which could potentiate the systemic BDNF might benefit the treatment of depressive symptom in bipolar disorder. In addition, peripheral administration of BDNF could increase hippocampal neurogenesis in adult mice and produces anti-depression effects (Schmidt and Duman, 2010). Hippocampal neuronal degeneration had been highly correlated to depression-like behavior. Thus, the down regulation of peripheral BDNF might represent the decreased neuronal protective ability in bipolar disorder. However, the mechanism of peripheral BDNF level in depression or related neuronal protective effects should be further studied.

\subsection{BDNF levels and schizophrenia}

We found that, in patients with schizophrenia, plasma BDNF levels were significantly lower than in healthy controls and patients with bipolar disorder, and that they were significantly negatively correlated with the negative symptoms. Schizophrenia is a complex and severe brain disorder that causes diverse disturbances in cognition, reality testing, mood, interpersonal relations, and work functions. Some quantitative abnormalities in the brain structures of patients with schizophrenia have been reported, e.g., enlarged ventricles, decreased cerebral and temporal lobe volume, and thalamic anomalies (Galderisi et al., 2008). Moreover, accumulating evidence has indicated an association between BDNF and schizophrenia. Reduced BDNF concentrations in the serum (Toyooka et al., 2002, Chen da et al., 2009), plasma (Buckley et al., 2007b), cerebral spinal fluid (Pillai et al., 2010), dorsolateral frontal cortex (Weickert et al., 2003), anterior cingulated cortex (Iritani et al., 2003), and hippocampus (Durany et al., 2001) were found in patients with schizophrenia. BDNF is known to modulate dopaminergic, GABAergic, and serotonergic receptors and regulate the synaptic transmission and plasticity in adult neuronal synapses (Alderson et al., 1990, Hyman et al., 1991, Daftary et al., 2012). Thus, BDNF dysregulation might be involved in the alteration of neuronal functions and lead to increase susceptibility to schizophrenia-like symptoms (Buckley et al., 2007a).

Although other studies had reported contradictory findings on BDNF expression in patients with schizophrenia. Takahashi et al. (Takahashi et al., 2000) reported elevated BDNF levels in the anterior cingulated cortex and hippocampus in 10 postmortem brains of patients with schizophrenia. Our findings agreed with those of Toyooka et al. (Toyooka et al., 2002), Pillai et al. (Pillai et al., 2010), and Buckley et al. (Buckley et al., 2007b), who reported significantly lower peripheral BDNF levels in patients with schizophrenia. Reduced peripheral BDNF levels were assumed to reflect the decrease of brain BDNF activity and may be used as an index of neuroplasticity status that underlies the pathophysiology of psychosis (Pillai et al., 2010). Our results resembled the previous study that peripheral BDNF levels were negative correlated to the negative symptoms of schizophrenia (Sotiropoulou et al., 2013). However, we did not find significant correlation between plasma BDNF and positive symptoms of schizophrenia, which is in accordance with previous studies (Chen da et al., 2009, Pillai et al., 2010). Inferred from previous findings, we propose that lower plasma BDNF level, an index of poorer neuroplasticity, might play an important role in the progression of negative symptoms. We hypothesize that BDNF dysregulation in the peripheral might lead contribute to central nervous systems BDNF expression and cause 
permanent alterations in brain function and leads to an increased risk of developing negative symptoms in schizophrenia. If organic mental illness and neurological disorder are ruled out, the plasma BDNF level might be one biological marker of negative symptom in schizophrenia.

\subsection{The relationship of plasma BDNF levels to BDNF Val66Met gene variants in mental illness}

To our knowledge, this is the first investigation that compares plasma BDNF levels with the $B D N F$ Val66Met SNP within different mental disorders. In this study, the distribution of $B D N F$ Val66Met SNP genotypes was not different in healthy control and patients with mental illness, which is in accordance with previous studies (Zhang et al., 2012, Sotiropoulou et al., 2013). In addition, we found no significant differences in plasma BDNF levels in different $B D N F$ Val66Met SNP genotypes in all of groups, which indicated that the $B D N F$ Val66Met gene polymorphism did not influence the plasma BDNF level in healthy humans and patients with bipolar disorder and schizophrenia. Our data resembled the previous study that revealed no significant difference in circulating BDNF levels between genotype subgroups of the BDNF Val66Met polymorphism in healthy control or patients with schizophrenia (Zhang et al., 2012). When stratified by the BDNF Val66Met genotypes, significant lower plasma BDNF level was found in the group of patients with schizophrenia compared to healthy control, which is consistent with the findings of Zhang et al. (2012). The plasma BDNF level seems to depend more on diagnostic effect than genetic effect. However, our data were different from Sotriopoulou et al. (Sotiropoulou et al., 2013) who studied a total of 50 patients with schizophrenia and found that the Met alelle carriers had lower circulating BDNF level and higher scores in the PANSS-Negative scale. In the current study, we include more patients $(n=151)$ with schizophrenia than the study by Sotriopoulou et al. (2013); we found the plasma BDNF levels and PANSS scores were not significantly different between the Met and Val carriers. Thus, we hypothesized that the down regulation of plasma BDNF in patients with schizophrenia does not depend on the BDNF Val66Met $S N P$ genotype, but on the degree of progression and pathology of illness in Han Chinese people.

Our study has some limitations. Although plasma BDNF might pass through the blood brain barrier and might be correlated with brain BDNF expression, the exact level of brain BDNF expression and its effect on different mental illnesses needs further study to draw stronger conclusions. Spinal cerebral fluid BDNF levels might be required to confirm our hypotheses and findings. However, for ethical reasons, we could not collect spinal cerebral fluid from most of our patients. Noninvasive methods of measuring brain BDNF and brain function need to be developed for more in-depth studies.

\subsection{Conclusion}

In conclusion, we found different plasma BDNF expression profiles in patients with BP-I, BP-II, and schizophrenia. The level of plasma BDNF was not affected by the $B D N F$ Val66Met variants, but by the process of the mental illness itself. The down regulation of plasma BDNF might indicate less neuronal protection and a certain degree of neuronal degeneration in mental illness.

Prog Neuropsychopharmacol Biol Psychiatry. Author manuscript; available in PMC 2020 April 06. 


\section{Acknowledgments}

This study was supported in part by grants DOH95-TD-I-111-004 and DOH98-TD-I-111-DD004 from the Taiwan Department of Health; grants NSC 98-2627-B-006-017, NSC98-2627-B-006-017, and NSC101-2314-B-006-063MY3 from the Taiwan National Science Council (to RBL); and by a grant from the National Cheng Kung University Project to Promote Academic Excellence and Develop a World Class Research Center.

We thank Ms Mei-Chun Wen, Pe-Ting Huang, Pin-His Yeh, and Hon-I Cheng for administrative assistance.

\section{Abbreviations}

BDNF

BP

BP-I

BP-II

SNP brain-derived neurotrophic factor

bipolar disorder

bipolar I disorder

bipolar II disorder

single nucleotide polymorphism

\section{Reference}

Alderson RF, Alterman AL, Barde YA, Lindsay RM (1990) Brain-derived neurotrophic factor increases survival and differentiated functions of rat septal cholinergic neurons in culture. Neuron 5:297-306. [PubMed: 2169269]

Benazzi F, Akiskal H (2006) The duration of hypomania in bipolar-II disorder in private practice: methodology and validation. Journal of affective disorders 96:189-196. [PubMed: 16427136]

Berman JA, Talmage DA, Role LW (2007) Cholinergic circuits and signaling in the pathophysiology of schizophrenia. Int Rev Neurobiol 78:193-223. [PubMed: 17349862]

Brunoni AR, Lopes M, Fregni F (2008) A systematic review and meta-analysis of clinical studies on major depression and BDNF levels: implications for the role of neuroplasticity in depression. The international journal of neuropsychopharmacology / official scientific journal of the Collegium Internationale Neuropsychopharmacologicum 11:1169-1180.

Buckley PF, Mahadik S, Pillai A, Terry A Jr. (2007a) Neurotrophins and schizophrenia. Schizophrenia research 94:1-11. [PubMed: 17524622]

Buckley PF, Pillai A, Evans D, Stirewalt E, Mahadik S (2007b) Brain derived neurotropic factor in first-episode psychosis. Schizophrenia research 91:1-5. [PubMed: 17306505]

Chen da C, Wang J, Wang B, Yang SC, Zhang CX, Zheng YL, Li YL, Wang N, Yang KB, Xiu MH, Kosten TR, Zhang XY (2009) Decreased levels of serum brain-derived neurotrophic factor in drugnaive first-episode schizophrenia: relationship to clinical phenotypes. Psychopharmacology 207:375-380. [PubMed: 19787338]

Cirulli F, Francia N, Branchi I, Antonucci MT, Aloe L, Suomi SJ, Alleva E (2009) Changes in plasma levels of BDNF and NGF reveal a gender-selective vulnerability to early adversity in rhesus macaques. Psychoneuroendocrinology 34:172-180. [PubMed: 18849121]

Cunha AB, Frey BN, Andreazza AC, Goi JD, Rosa AR, Goncalves CA, Santin A, Kapczinski F (2006) Serum brain-derived neurotrophic factor is decreased in bipolar disorder during depressive and manic episodes. Neuroscience letters 398:215-219. [PubMed: 16480819]

Daftary SS, Calderon G, Rios M (2012) Essential role of brain-derived neurotrophic factor in the regulation of serotonin transmission in the basolateral amygdala. Neuroscience 224:125-134. [PubMed: 22917617]

Dilsaver SC (1986) Cholinergic mechanisms in affective disorders. Future directions for investigation. Acta Psychiatr Scand 74:312-334. [PubMed: 2949489] 
Driscoll I, Martin B, An Y, Maudsley S, Ferrucci L, Mattson MP, Resnick SM (2012) Plasma BDNF is associated with age-related white matter atrophy but not with cognitive function in older, nondemented adults. PloS one 7:e35217. [PubMed: 22523577]

Dunner D (Goodnick P, Editor. 1998) Diagnostic revision for DSM-IV, in Mania, Clinical and Research Perspectives. American Psychiatric Press p. 3-10.

Durany N, Michel T, Zochling R, Boissl KW, Cruz-Sanchez FF, Riederer P, Thome J (2001) Brainderived neurotrophic factor and neurotrophin 3 in schizophrenic psychoses. Schizophrenia research 52:79-86. [PubMed: 11595394]

Egan MF, Kojima M, Callicott JH, Goldberg TE, Kolachana BS, Bertolino A, Zaitsev E, Gold B, Goldman D, Dean M, Lu B, Weinberger DR (2003) The BDNF val66met polymorphism affects activity-dependent secretion of BDNF and human memory and hippocampal function. Cell 112:257-269. [PubMed: 12553913]

Endicott J, Spitzer RL (1978) A diagnostic interview: the schedule for affective disorders and schizophrenia. Archives of general psychiatry 35:837-844. [PubMed: 678037]

Fan J, Sklar P (2008) Genetics of bipolar disorder: focus on BDNF Val66Met polymorphism. Novartis Foundation symposium 289:60-72; discussion 72-63, 87-93. [PubMed: 18497095]

Fernandes BS, Gama CS, Cereser KM, Yatham LN, Fries GR, Colpo G, de Lucena D, Kunz M, Gomes FA, Kapczinski F (2011) Brain-derived neurotrophic factor as a state-marker of mood episodes in bipolar disorders: a systematic review and meta-regression analysis. Journal of psychiatric research 45:995-1004. [PubMed: 21550050]

Galderisi S, Quarantelli M, Volpe U, Mucci A, Cassano GB, Invernizzi G, Rossi A, Vita A, Pini S, Cassano P, Daneluzzo E, De Peri L, Stratta P, Brunetti A, Maj M (2008) Patterns of structural MRI abnormalities in deficit and nondeficit schizophrenia. Schizophrenia Bulletin 34:393-401. [PubMed: 17728266]

Gama CS, Andreazza AC, Kunz M, Berk M, Belmonte-de-Abreu PS, Kapczinski F (2007) Serum levels of brain-derived neurotrophic factor in patients with schizophrenia and bipolar disorder. Neuroscience letters 420:45-48. [PubMed: 17442489]

Gratacos M, Gonzalez JR, Mercader JM, de Cid R, Urretavizcaya M, Estivill X (2007) Brain-derived neurotrophic factor Val66Met and psychiatric disorders: meta-analysis of case-control studies confirm association to substance-related disorders, eating disorders, and schizophrenia. Biological psychiatry 61:911-922. [PubMed: 17217930]

Hashim HM, Fawzy N, Fawzi MM, Karam RA (2012) Brain-derived neurotrophic factor Val66Met polymorphism and obsessive-compulsive symptoms in Egyptian schizophrenia patients. Journal of psychiatric research 46:762-766. [PubMed: 22521161]

Huang SY, Lin WW, Ko HC, Lee JF, Wang TJ, Chou YH, Yin SJ, Lu RB (2004) Possible interaction of alcohol dehydrogenase and aldehyde dehydrogenase genes with the dopamine D2 receptor gene in anxiety-depressive alcohol dependence. Alcoholism, clinical and experimental research 28:374384.

Hyman C, Hofer M, Barde YA, Juhasz M, Yancopoulos GD, Squinto SP, Lindsay RM (1991) BDNF is a neurotrophic factor for dopaminergic neurons of the substantia nigra. Nature 350:230-232. [PubMed: 2005978]

Iritani S, Niizato K, Nawa H, Ikeda K, Emson PC (2003) Immunohistochemical study of brain-derived neurotrophic factor and its receptor, TrkB, in the hippocampal formation of schizophrenic brains. Prog Neuropsychopharmacol Biol Psychiatry 27:801-807. [PubMed: 12921913]

Kanazawa T, Glatt SJ, Kia-Keating B, Yoneda H, Tsuang MT (2007) Meta-analysis reveals no association of the Val66Met polymorphism of brain-derived neurotrophic factor with either schizophrenia or bipolar disorder. Psychiatric genetics 17:165-170. [PubMed: 17417060]

Karege F, Schwald M, Cisse M (2002) Postnatal developmental profile of brain-derived neurotrophic factor in rat brain and platelets. Neuroscience letters 328:261-264. [PubMed: 12147321]

Laske C, Stransky E, Leyhe T, Eschweiler GW, Wittorf A, Richartz E, Bartels M, Buchkremer G, Schott K (2006) Stage-dependent BDNF serum concentrations in Alzheimer's disease. J Neural Transm 113:1217-1224. [PubMed: 16362629] 
Lu W, Zhang C, Yi Z, Li Z, Wu Z, Fang Y (2012) Association between BDNF Val66Met polymorphism and cognitive performance in antipsychotic-naive patients with schizophrenia. Journal of molecular neuroscience : MN 47:505-510. [PubMed: 22477643]

Mizuno K, Carnahan J, Nawa H (1994) Brain-derived neurotrophic factor promotes differentiation of striatal GABAergic neurons. Developmental biology 165:243-256. [PubMed: 8088442]

Monteleone P, Serritella C, Martiadis V, Maj M (2008) Decreased levels of serum brain-derived neurotrophic factor in both depressed and euthymic patients with unipolar depression and in euthymic patients with bipolar I and II disorders. Bipolar disorders 10:95-100. [PubMed: 18199246]

Ohira K, Hayashi M (2009) A New Aspect of the TrkB Signaling Pathway in Neural Plasticity. Current neuropharmacology 7:276-285. [PubMed: 20514207]

Pan W, Banks WA, Fasold MB, Bluth J, Kastin AJ (1998) Transport of brain-derived neurotrophic factor across the blood-brain barrier. Neuropharmacology 37:1553-1561. [PubMed: 9886678]

Pillai A, Kale A, Joshi S, Naphade N, Raju MS, Nasrallah H, Mahadik SP (2010) Decreased BDNF levels in CSF of drug-naive first-episode psychotic subjects: correlation with plasma BDNF and psychopathology. The international journal of neuropsychopharmacology / official scientific journal of the Collegium Internationale Neuropsychopharmacologicum 13:535-539.

Schmidt HD, Duman RS (2010) Peripheral BDNF produces antidepressant-like effects in cellular and behavioral models. Neuropsychopharmacology : official publication of the American College of Neuropsychopharmacology 35:2378-2391. [PubMed: 20686454]

Shimizu E, Hashimoto K, Watanabe H, Komatsu N, Okamura N, Koike K, Shinoda N, Nakazato M, Kumakiri C, Okada S, Iyo M (2003) Serum brain-derived neurotrophic factor (BDNF) levels in schizophrenia are indistinguishable from controls. Neuroscience letters 351:111-114. [PubMed: 14583394]

Sotiropoulou M, Mantas C, Bozidis P, Marselos M, Mavreas V, Hyphantis T, Antoniou K (2013) BDNF serum concentrations in first psychotic episode drug-naive schizophrenic patients: associations with personality and BDNF Val66Met polymorphism. Life sciences 92:305-310. [PubMed: 23333821]

Spalletta G, Morris DW, Angelucci F, Rubino IA, Spoletini I, Bria P, Martinotti G, Siracusano A, Bonaviri G, Bernardini S, Caltagirone C, Bossu P, Donohoe G, Gill M, Corvin AP (2010) BDNF Val66Met polymorphism is associated with aggressive behavior in schizophrenia. European psychiatry : the journal of the Association of European Psychiatrists 25:311-313. [PubMed: 20430595]

Takahashi M, Shirakawa O, Toyooka K, Kitamura N, Hashimoto T, Maeda K, Koizumi S, Wakabayashi K, Takahashi H, Someya T, Nawa H (2000) Abnormal expression of brain-derived neurotrophic factor and its receptor in the corticolimbic system of schizophrenic patients. Molecular psychiatry 5:293-300. [PubMed: 10889532]

Teh CA, Lee TS, Kuchibhatla M, Ashley-Koch A, Macfall J, Krishnan R, Beyer J (2012) Bipolar disorder, brain-derived neurotrophic factor (BDNF) Val66Met polymorphism and brain morphology. PloS one 7:e38469. [PubMed: 22859933]

Terracciano A, Lobina M, Piras MG, Mulas A, Cannas A, Meirelles O, Sutin AR, Zonderman AB, Uda M, Crisponi L, Schlessinger D (2011) Neuroticism, depressive symptoms, and serum BDNF. Psychosomatic medicine 73:638-642. [PubMed: 21949427]

Toyooka K, Asama K, Watanabe Y, Muratake T, Takahashi M, Someya T, Nawa H (2002) Decreased levels of brain-derived neurotrophic factor in serum of chronic schizophrenic patients. Psychiatry research 110:249-257. [PubMed: 12127475]

Webster MJ, Weickert CS, Herman MM, Kleinman JE (2002) BDNF mRNA expression during postnatal development, maturation and aging of the human prefrontal cortex. Brain research 139:139-150. [PubMed: 12480128]

Weickert CS, Hyde TM, Lipska BK, Herman MM, Weinberger DR, Kleinman JE (2003) Reduced brain-derived neurotrophic factor in prefrontal cortex of patients with schizophrenia. Molecular psychiatry 8:592-610. [PubMed: 12851636]

Whalley HC, Baig BJ, Hall J, Job DE, McIntosh AM, Cunningham-Owens DG, Johnstone EC, Lawrie SM (2010) Effects of the BDNF val66met polymorphism on prefrontal brain function in a 
population at high genetic risk of schizophrenia. American journal of medical genetics Part B, Neuropsychiatric genetics : the official publication of the International Society of Psychiatric Genetics 153B:1474-1482.

Zhang XY, Chen da C, Xiu MH, Haile CN, Luo X, Xu K, Zhang HP, Zuo L, Zhang Z, Zhang X, Kosten TA, Kosten TR (2012) Cognitive and serum BDNF correlates of BDNF Val66Met gene polymorphism in patients with schizophrenia and normal controls. Human genetics 131:11871195. [PubMed: 22362486] 


\section{Highlights}

- The $B D N F$ Val66Met polymorphism in Han Chinese patients with different mental illness

- The plasma BDNF levels in Han Chinese patients varied with different mental illness.

- $\quad$ Plasma BDNF in mental disorders is affected by the process and progression of illness 


\section{를}

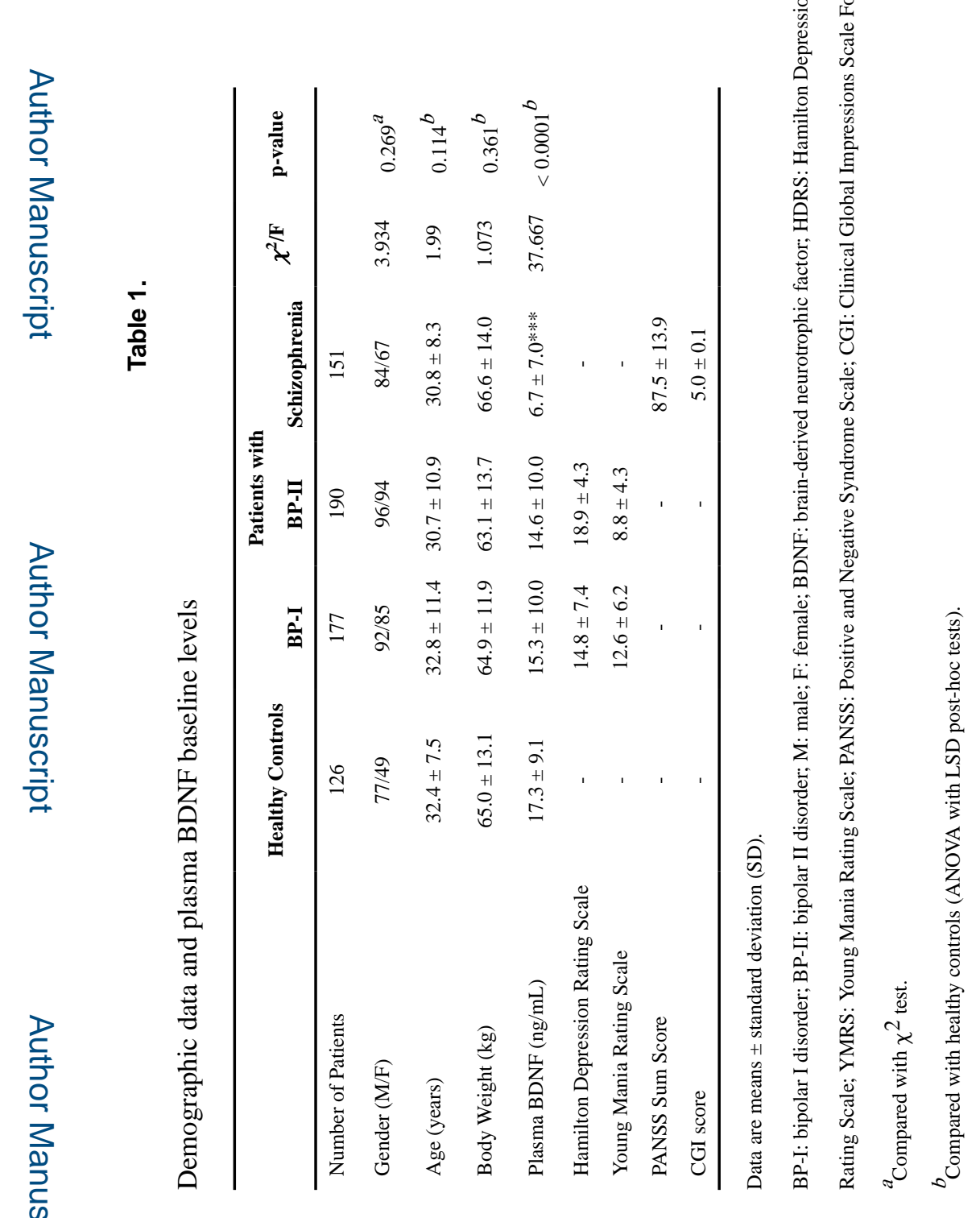

Prog Neuropsychopharmacol Biol Psychiatry. Author manuscript; available in PMC 2020 April 06. 


\section{를 \\ ํㅗㄹ}

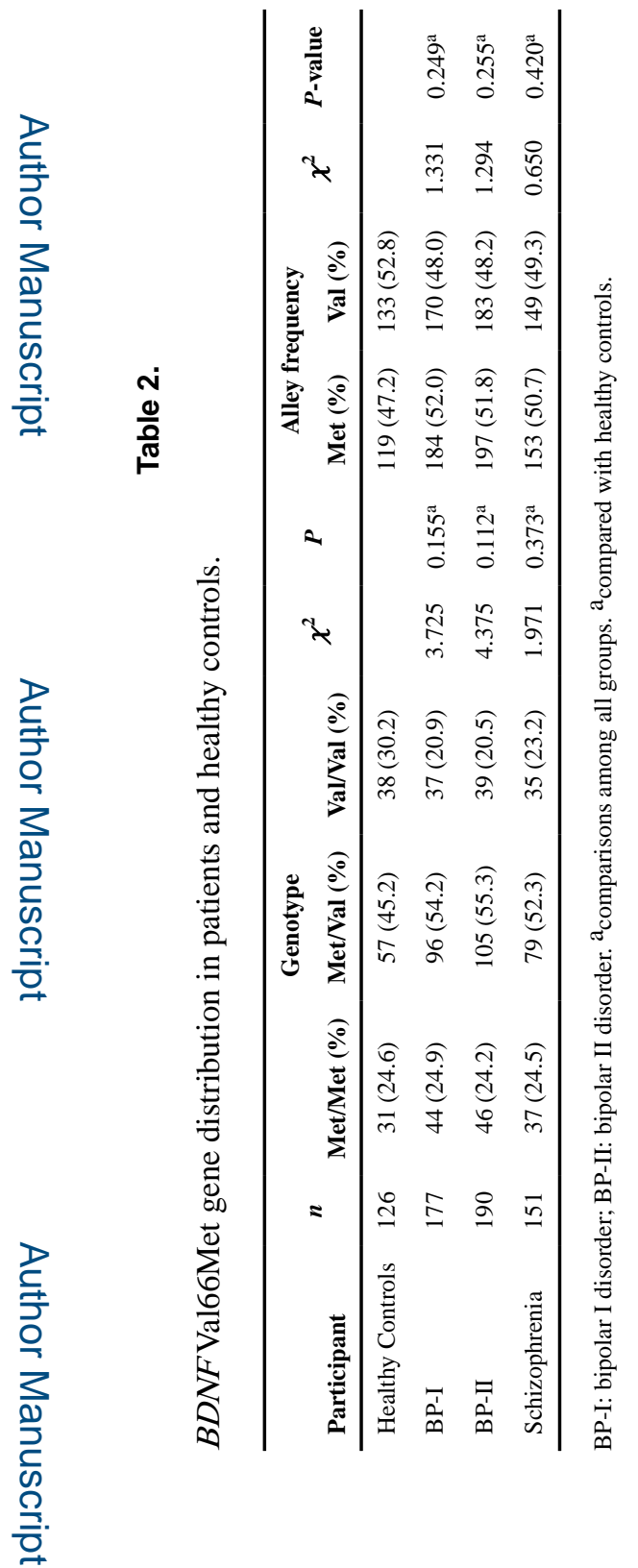

Prog Neuropsychopharmacol Biol Psychiatry. Author manuscript; available in PMC 2020 April 06. 


\section{롤 \\ 일}
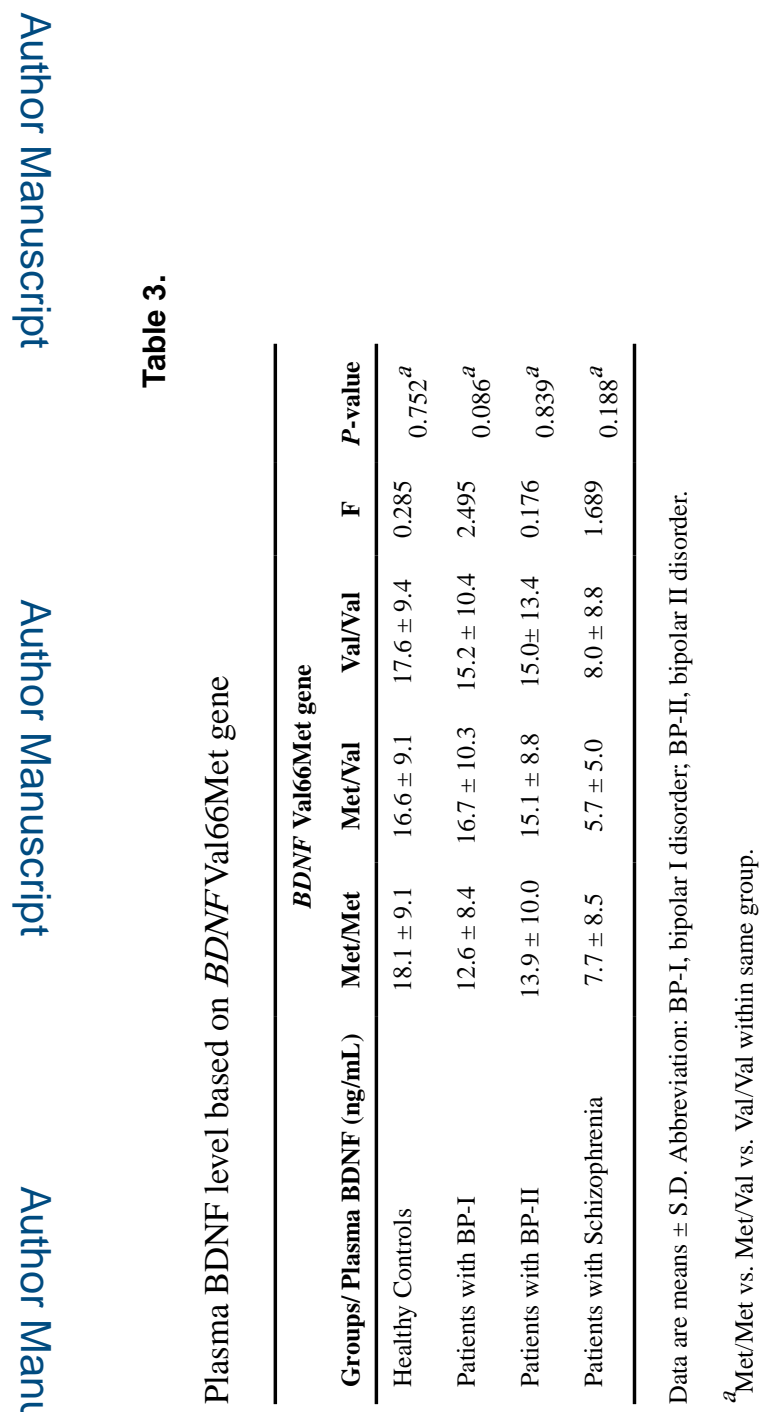

Prog Neuropsychopharmacol Biol Psychiatry. Author manuscript; available in PMC 2020 April 06. 